\title{
Efficiency of Optical Fiber Communication for Dissemination of Information within the Power System Network.
}

\author{
Engr Dr Mrs G.N Ezeh ${ }^{1}$ Okwe Gerald Ibe ${ }^{2}$ \\ 1.Senior lecturer, Department of Electrical/Electronic Engineering Federal University of Technology Owerri \\ Imo State (Nigeria) \\ 2. Student Department of Electrical/Electronic Engineering Federal University of Technology Owerri Imo State
}

(Nigeria)

\begin{abstract}
Within the power system network, consistent domestic communications are essential to ensure safety, security and control of the power system equipments. Such communications customarily have been provided by methods such as power line carrier and microwave radio systems but are more recently being supplemented or replaced by Fiber optics. This paper focuses on the practical steps to review and evaluation on the effectiveness of using Fiber optic cable technology in the domestic communication of power system network. With the advent of information and communication technology, it has become obvious that Fiber optic is replacing this crude method of data communication. It offers a unique solution to ever increasing demand for bandwidth because of its remarkably high capacity for carrying data, and guaranteed consistency of signal transmission over the entire transmission network. A pair of Fiber has the ability to carry over eight thousand simultaneously voice channels and has high immunity to electromagnetic interference. All these advantages made it extra-ordinarily useful in data communication like Internet, multimedia and scada applications. Over short or long distances, video, audio and data signals arrive at their destination in the same perfect quality as they originated and also assures security of data being transmitted.
\end{abstract}

Keywords: Optical Fibre, Security, attenuation, Fiber degradation, bandwidth, splicing, Scada.

Accepted Date: 08 Feb 2013

\section{Introduction}

The explosive growth of information technology and broadband applications has been driving the strong demand for bandwidth in the telecommunications networks. Enabled by present Optical Fiber communication technology, Fiber Optics is emerging as the fastest and most cost-effective way to maximize and expand network capacity. In the last five years, the Fiber optic communications industry has experienced an unprecedented growth and rapid technological changes. Power companies are one of the biggest users of communications. This is because reliable internal communications are crucial in Power Company to ensure protection and control of the power system equipments. If there is a poor communication path, there could be false information or no information transmitted from the control centre that informs the operators and consumers about the status of their equipment in the network. The computers (servers) that controls the application software are stored in the control room at the Power central office locations or headquarters. With some of these applications, the operators may offer to host the software equipment at their site or a remote site in which the utility will have access to obtain their information. This allows the operators to maintain the servers and support all the software applications such that the application runs properly. This communication technique offers a variety of advantages that are suitable for electrical companies to disseminate information within their network. Such communications originally have been provided by methods such as power line carrier and microwave radio systems but are more recently being supplemented by Fiber Optics technology. However, with the advent of digital fault protection systems, integrated power system automation signal densities are increasing and Fiber Optic communication can offer a unique solution to the ever increasing demand in the future. Consequently, many electric companies are installing high capacity Fiber Optic cables and wires on their high voltage lines to satisfy their own internal communication needs. Power companies that have this backbone will gain additional proceeds, by providing excess capacity to telecommunication network providers if they want. Overhead installation of this cables provide the company with cost-effective alternative routes and at the same time benefit them by using existing facilities like electric poles and towers. The inherent advantage of Fiber Optic technology as a means of communication is that it provides fixed link, point to point communications with a remarkably high capacity for carrying data. For example, a single pair of fibers can carry nearly eight thousand simultaneous video and voice channels. The immunity of Fiber Optics to electromagnetic interference is another advantage for its use in power delivery systems as long as care is taken 
to shield any terminal and repeater stations. Typically, there are no radiation or Frequency assignment difficulties as commonly experienced with power line carrier, intra-bundle, and microwave communication systems. Also, Fiber Optic increases the security of the transmission systems since the technology virtually eliminates the unauthorized monitoring of vital communication. Fiber Optic does not require coupling devices or other specialized connectors and can be easily and cost-effectively integrated into any digital network.

\subsection{Methods Used In Overhead Distribution Of Fiber Cable}

ADSS (All-Dielectric Self-Supporting) is the simplest concept for aerial Fiber-optic cable: it is an underground Fiber Optic cable made stronger to allow it to be installed by attaching it to a series of poles. The cable needs to be physically strong because it will be supported only at each pole along the route and will have to support its own weight across the half-span on each side of the pole. This is in contrast to an underground cable which is fully supported inside a duct or in a back-filled trench along its whole length. In addition to its own weight, ADSS cable must support the extra loads imposed by wind pressure and by the build up of ice when this is problem in exposed locations. These extra loads can be significant and require carefully designed clamps to spread the mechanical strain over several meters of cable at each pole to prevent any risk of damage. ADSS cables have the advantage that they are completely independent of the electricity supply network, even though they are installed on the same poles. Potentially the two networks can be owned, managed and maintained by different organisations, although there are safety issues when people carry out installation and maintenance work in close proximity to live electricity conductors.

OPPC (Optical Phase Conductor). This is a replacement electrical conductor that has Optical Fibers built into it as part of the manufacturing process. The Fibers are inside the conductor, usually contained within a stainless steel tube. OPPC is installed on an overhead electricity line in place of one of the normal conductors.OPPC replaces one of the normal conductors and therefore it adds nothing to the appearance of an overhead line and it does not affect the mechanical or electrical rating of the line. From this point of view, OPPC is the least obvious and most secure of all of the cable types. However it is also the technology that is most intimately associated with the electricity supply network as it physically forms part of this network. Any maintenance activity on either the communications or power network involving OPPC will have an impact on the operations of both networks.OPPC is normally only installed as part of the construction of a new line or during the complete refurbishment of an existing line and so it is unlikely that OPPC will be specified by any organisation other than a power company.

AccessWrap: This is a technique that installs a Fiber-Optic cable onto an overhead electricity distribution line by wrapping it securely onto one of the power conductors. This is a scaled down version of the SkyWrap process that has been used since early 80 s to install Fiber-Optic cables onto power transmission lines; the smaller, lighter AccessWrap machine is designed to work on power lines supported by wood or concrete poles and with conductors spaced only $0.5 \mathrm{~m}$ apart. The optical cable is supported by its host conductor and so it does not need to carry any of its own weight. Therefore it can be very small and this means it has little effect on the mechanical and electrical performance of the overhead line; it also has little impact on the appearance of the line. Installation is carried out using a special device which travels along the host conductor carrying a drum of Optical Fibre cable. The device rotates as it moves and wraps the cable under carefully controlled tension onto the host conductor at a pitch length of about three quarters of a metre. Clamps are used on each side of each pole to hold the cable in place on the conductor. The machine moves at about walking pace with about 15 minutes required at each pole to lift the machine onto the next span and put the clamps in place. AccessWrap does not place extra load on the poles supporting the power line nor does it reduce the ground clearance under the line and these are major advantages over ADSS in some circumstances. These products create an opportunity for power companies to roll out communications networks on to their electricity distribution infrastructure, potentially connecting all the way through to the users' premises and linking these to head ends at major substations or regional control centres. This type of infrastructure may be required to provide the communication networks to support Smart Grids, and power companies may build these networks for this purpose only. However, once built, such networks would support other applications and could generate revenue opportunities in providing carrier services to third parties such as broadband service providers and mobile operators.

\subsection{Advantages}

The key advantages of using overhead electricity distribution lines to carry cables providing broadband connectivity can be summarised in three distinct areas: speed, security and cost

- Speed: It is always much, much quicker to install Fiber-Optic cable by attaching it to poles than it is to dig trenches to bury it underground. Directional drilling or ploughing are alternative ways of installing 
underground cable, but these are also slow and expensive compared to installation on overhead lines. Circumstances will vary according to the time of year with factors including weather conditions, whether or not crops or animals are in the fields and what the ground conditions are like underfoot; however it is generally possible to install at least $1 \mathrm{~km}$ of Fibre Optic cable a day on overhead power lines and up to $5 \mathrm{~km}$ per day is possible in favorable circumstances.

- Security: This is a key concern in any Fiber-Optic cable installation. Cables have been installed on overhead power lines since the very early 1980s and have developed an excellent reputation for security and reliability over that time. Power utilities use these cables to carry critical communications for control of the electricity network. Fiber-optic cables installed above ground are not subject to 'dig-ups' which is the biggest cause of cable damage that are being experienced by companies everytime. Cables that are installed as part of the electricity infrastructure are protected by the proximity of power conductors, which provide protection against theft and vandalism.

- Cost: The higher unit cost of aerial cables compared to underground cables is more than offset by the much lower cost of installation and therefore aerial cables have the lowest total cost. Aerial cables have much higher installation rates and so networks are built much more quickly, begin to provide services earlier and so have quicker returns on investment. Put another way, with reduced initial costs and earlier in-service dates, aerial cables have shorter pay back times than underground networks

\section{Literature Review}

The fastest means of carrying information would be the use of fiber optic networks. This offers the transmission of data from one location to another. Fiber optic is an optical tube cable that is designed to transport data threw glass over an optical light. An optical light has the capability to travel at a distance of 126,000 miles per second within an optical fiber. There are many components that make up the fiber optic cable. The first section of the cable is the optical fibers. The optical fiber is the component that actually transports the data in the fiber optic cable. The optical fiber component is made up with three different parts: the buffer coating, the cladding, and the core. The buffer coating, which is the outer portion, provides the cable its strength and support in that helps prevent the cable from breaking. The cladding and the core both are designed to help enhance the transmission of the optical signal. The second section of the fiber is designed for the outdoor environment. Many cables have different designs, but for most cables today they are designed with a Kevlar portion that helps add extra strength to the cable. To support the optical fibers inside the cable there is an outer sheath coating that is a made of an extremely hard plastic material. This is to help the bending radius of the cable and add support through the lifetime of the cable. Fiber optics can operate in the range of $850 \mathrm{~nm}$ (nanometers), $1300 \mathrm{~nm}$, or $1550 \mathrm{~nm}$ wavelengths for data transmission.

\subsection{THREE BASIC PARTS OF FIBRE-OPTIC SYSTEM:}

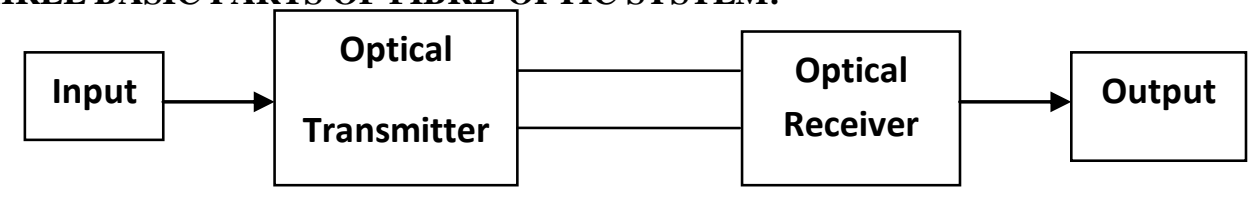

Fig 1 Generic Optic communication system

Transmitter: The transmitter unit converts an electrical signal to an optical signal. The light source is typically a light-emitting diode, LED, or a laser diode. The light source performs the actual conversion from an electrical signal to an optical signal. The driving circuit for the light source changes the electrical signal into the driving current.

- Fiber-optic cable: The fiber-optic cable is the transmission medium for carrying the light. The cable includes the Optical Fibers in their protective jacket.

- Receiver: The receiver accepts the light or photons and converts them back into an electrical signal. In most cases, the resulting electrical signal is identical to the original signal fed into the transmitter. There are two basic sections of a receiver. First is the detector that converts the optical signal back into an electrical signal. The second section is the output circuit, which reshapes and rebuilds the original signal before passing it to the output. Depending on the application, the transmitter and receiver circuitry can be very simple or quite complex. Other components that make up a fibre-optic transmission system, such as couplers, multiplexers, optical amplifiers, and optical switches, provide the means for building more complex links and communications networks. The transmitter, fiber, and receiver, however, are the basic elements in every Fiber-Optic system. Beyond the simple link, the Fibre-Optic medium is the fundamental building block for Optical communications. Most electrical signals can be transported optically. Many optical components have been invented to permit 
signals to be processed optically without electrical conversion. Indeed, one goal of optical communications is to be able to operate entirely in the optical domain from system end to end

\subsection{Two Types Fiber Optic Cables}

There are 2 different types of fiber optic cables that are used within fiber optic networks. One of these is the single-mode fiber and the other is the multi-mode fiber cable. The single-mode fiber carries an enormous amount of information in one direction. Single-mode fiber is used if there needs to be no return path for the communication network. For instance, a return path would be used if data is to be transmitted and received in data transmission. The case for accessing and using the Internet, the data needs to travel in both directions of downstream and upstream. The multi-mode fiber can travel in more than one direction. Multi-mode fiber is mainly used where a signal needs to be transmitted and received from multiple locations. Many applications of multi-mode fiber are used within networks in industrial or commercial buildings and around college institutions to allow for high data transmission rates.

Fiber optic networks are not different from any other network in the sense that it offers opportunities for failure. Fiber optics have many other applications that makes fiber optics very versatile. When running communications like a DSL line into a substation, the provider's ground potential and the substation ground potential might be at different levels. This can cause failures with equipment inside the substation or the communication company's equipment. For this reason, many electrical companies require the two grounding systems to be isolated. One way to do this is with fiber optics. An Ethernet cable is used when a data signal exits or enters the modem for data transmission. This media of Ethernet can be converted into a fiber signal and then transported into the substation with no worries about the two grounding potentials. This is a form of isolation.

\subsection{Types Of Failures Associated With Fiber Network}

The failure classes that are associated with fiber optic networks are classified as patch panel failures, installation failures, and construction failures. Patch panel failures are failures that create malfunctions in the system by high attenuation. Poor connection points can cause some of these malfunctions when the fiber was terminated with the appropriate connectors. When the fiber is spliced together poorly the signal inside the fiber optic cable can have large dB losses. If the connections were not inserted in the connectors correctly or the fiber cable has fractured parts in the glass, there can be very high attenuation to no signal propagation into other parts of the communication network. This is going to possibly show communication outages within the system. Installation failures are associated with failures that are caused when installing the fiber optic network. If a fiber optic cable is bent past the specification bending radius of the cable then the cable can fail instantly or could possible fail over time. This might not be an automatic failure as mentioned but could be failures later in time as the fiber optics weaken. This mainly happens when the installer is not aware of the specification of the cable and not paying attention to what he or she is doing. Failures in fiber optics can also be caused by improper dressing and from terminating the fiber optic connections. This kind of installation failure is sort of an overlap from a patch panel failure. When fiber optic cable connections are terminated to the cable poorly, weather it be ST connectors, SC connectors or similar connectors for example, these cables can present a point of failure in the installation process. Terminating fiber optic cable can be a very hard skill to master. The last failure class is the failures that are related to the construction of the fiber optics networks. These cables are always going to be strung from pole to pole, similar to the high or medium voltage lines that form our electric grid.

\subsection{Physical Phenomena That Cause Cable Failures:}

Corona discharge at the tips of assembly rods that are not all terminated at the same location on the cable (i.e., one rod sticks out further than others); and Dry band arcing on the surface of the cable under polluted conditions BPA concentrated their observations and experiences on the corona phenomena.

\subsubsection{Corona}

The outer plastic sheath of an ADSS aerial cable is subject to erosion by discharges when placed in the electric field of the phase conductors of the overhead power line. While the influence is negligible at lower voltage levels, problems have been observed on lines with higher voltage ratings. Typically, the surface of the ADSS cable sheath changes in appearance and structure near the dead end and armor grip suspension points. These changes indicated a degradation of the ADSS sheath surface. Typically, the damage of the sheath, normally black with high density polyethylene, arises within a few weeks to months. Consequently, the sheath material of ADSS aerial cables must be resistant to electrical discharges

\subsubsection{Grounding}

Since the ADSS used in high voltage environments does not contain metallic parts, there is no question of grounding the cable. However, it must be recognized that the ADSS aerial cable is mechanically attached to 
grounded parts at each structure. These grounded parts include the structure members, clamps and armor rods. Also, since the ADSS is placed in the electric field of the energized phase conductors, significant induced voltages may be developed on the cable. This is important from the viewpoint of ADSS cable aging and safety of personnel working in the cable while the line remains energized

\subsection{Advantages Of Fibre-Optic Communications}

In addition to Fiber optics technical advantages, the cost of materials for Fiber optics is becoming more attractive because the cost of copper wire has risen substantially in recent years.

\section{Longer Distances}

A significant benefit of fiber-optic transmission is the capability to transport signals long distances. Basic systems are capable of sending signals up to $5 \mathrm{~km}$ over multimode fiber and up to $80 \mathrm{~km}$ over single mode without repeaters. Most modern Fibre-optic systems transport information digitally. A digital fiber-optic system can be repeated or regenerated virtually indefinitely. An electro-optical repeater or an erbium doped fiber amplifier (EDFA) can be used to regenerate or amplify the optical signal.

\section{Multiple Signals}

As discussed in previous sections, fiber has a bandwidth of more than $70 \mathrm{GHz}$ using typical off-theshelf Fibre-optic transport equipment. Theoretically, hundreds, even thousands, of video and audio signals can be transported over a single fiber. This is achieved by using a combination of time-division multiplexing (TDM) and optical multiplexing. Fiber-optic transport equipment is readily available to transport more than 8 video and 32 audio channels per wavelength. Of the- shelf coarse wave-division multiplexing CWDM equipment easily provides up to 18 wavelengths. This combination of equipment provides up to 144 video and 576 audio channels.

\section{Size}

Fiber-optic cable is very small in diameter and size when compared to copper. A single strand of Fiber Optic cable is about $3 \mathrm{~mm}$. A video coaxial cable is typically much larger. Fiber cable facilitates higher capacity in building conduits. There is often limited space in existing building conduits for infrastructure expansion. In mobile and field productions for sports and news events, fiber is often the cable of choice due to space limitations in a mobile and electronic news gathering vehicle.

\section{Weight}

A fiber-optic cable is substantially lighter in weight than copper cable. A single core PVC-jacketed fiber weighs about 25 pounds per kilometer; RG-6 copper coaxial cable may be three to four times as much.

\section{Noise Immunity}

A signal traveling on a copper cable is susceptible to electromagnetic interference. In many applications it is unavoidable to have to route cabling near power substations; heating, ventilating, and airconditioning (HVAC) equipment; and other industrial sources of interference. A signal traveling as photons in an optical fiber is immune to such interference. The photons traveling down a fiber cable are immune to the effects of electromagnetic interference. In military applications, fiber systems are immune to an electromagnetic pulse (EMP) generated by a nuclear explosion in the Earth's atmosphere. Fiber-optic equipment is used in command and control bunkers to isolate facilities and systems from EMP interference. A fiber-optic signal does not radiate any interference or noise.

\section{Ease of Installation}

One of the myths regarding fiber is that it is difficult to install and maintain. This may have been true in the early days, but now it is as simple to terminate an optical fiber with a connector as it is to install a BNC connector on coax. Fiber-optic termination kits are now available that require no epoxy and special polishing. Simple cable stripping tools are used, similar to those used for copper coax, to prepare the fiber for termination. Epoxy-free connectors are available to terminate both multimode and single-mode fiber-optic cable. The connectors are already prepolished. No polishing equipment is needed.

\section{Low transmission loss}

The development of Optical Fibres over the last twenty years has resulted in production of Optical Fibre cables which exhibit very low attenuation or transmission loss in comparison with the best copper conductors. 


\section{Large bandwidth}

The optical carrier frequency yields a far greater potential transmission bandwidth than metallic cable systems. Information-carrying capacity of Optical Fibre system has proved far superior to the best copper cable systems. By comparison the losses in wideband coaxial cable systems restrict the transmission distance to only a few kilometers at bandwidths over one hundred megahertz.

\section{System reliability}

These features involves stem from the low loss property of Optical Fibre cables which reduces the requirement for intermediate repeaters to boost the transmitted signal strength. Hence with fewer repeaters, system reliability is generally enhanced in comparison with conventional electrical conductor systems. It also tend to reduce the maintenance time and cost.

\section{Low cost}

Optical Fibres offer potential for low cost line communication compared to those with copper conductors. Overall system cost when utilizing Optical Fibre communication on long-haul links is substantially less than those for equivalent electrical line systems.

\section{Electrical Modeling And Analysis}

Electric field modeling and analysis of OPGW and the WRAP Fiber optic cables is not typically required beyond traditional analysis normally performed for regular ground wires. However, in unusual situations in which ground wire corona occurs, WRAP aerial cable placement can be analyzed using a 2-D electrostatic analysis. Detailed 3-D modeling and analysis of electric field patterns near the structures are recommended in order to identify locations that will minimize the electric field induced degradation of ADSS aerial cable. Otherwise, catastrophic failures within a short period of time (2 to 10 months) can result. However, 3-D analyses require significant resources which may not always be available prior to placement of aerial optical cables and wires. At a minimum, a 2-D space potential calculation should be used to identify the most suitable placement location for standard and angle suspension structures. At the same time, it should be realized that a more rigorous 3-D analysis may allow the placement of ADSS cables in potentials up to $40 \mathrm{kV}$ (i.e., calculated in a 2-D analysis) by considering the shielding effect of the transmission structure.

\subsection{Fiber Preparation}

Fiber Striping: Optical Fibre must be stripped of buffer coatings to allow a closer fit within precision connectors. (Note always wear safety glasses or goggles when working directly with fibers)

Mechanical stripping: Buffer coatings are usually removed mechanically with sharp blades or calibrated stripping tools. In any type of mechanical stripping, the key is to avoid nicking the fiber.

Splicing optical fiber: Preparation of fibers for splicing is very similar to the process described under connecterization. After jacket materials, strength members and buffer tubes have been cut to the appropriate lengths, the fiber buffer coatings must be removed.

Cleaving: After the buffer coatings have been removed, fibers must be cleaved in preparation for splicing. Cleaving is a method of breaking a fiber in such a way as to create a smooth, square end on the fiber.

\section{Splicing methods}

There are two basic types of splices

1. Fusion Splicing: Fusion splices are made by positioning cleaned, cleaved fiber ends between two electrodes and applying an electric arc to fuse the ends together. A perfusion arc is applied to the fiber while the ends are still separated to vaporize volatile materials which cause bubbles. Final precise alignment is done by moving fiber ends together until there is slight pressure between end surfaces. An ideal fusion cycle is short, ramped cycle is short and uses ramped or gradually increasing arc currents. A short ramped cycle is considered least likely to produce excessive thermal stress in fibers. Cold temperatures is require increased time and arc current Experienced operators consistently produce fusion splices with loss less than $0.2 \mathrm{~dB}$ per splice and averaging $0.3 \mathrm{~dB}$ on multi mode fibers Sophiscated fusion splicing systems for single mode fibers produce typical splice losses 0.05 to $0.1 \mathrm{~dB}$.

2. Mechanical Splicing: This splicing systems positions fiber ends closely in retaining and aligning assemblies. Focusing and collimating lenses may be used to control and concentrate light that would otherwise escape. Index matching gels, fluids and adhesives are used to form a continuous optical path between fibers and reduce reflection losses. 


\subsection{Optical Power Measurement}

When optical fiber has been installed, all splices made and connectors attached, it must be determined if the system is capable of delivering the required power. The simplest test require light source of same type wavelength and approximate power as that equipment to be used. The system equipment itself is often satisfactory source. The first step is to obtain an approximate measure of system launch power. A short test cable with same fiber and connector style as the installed cable can be used for this procedure. One end of the short cable is connected to the light launching equipment. The other end is connected to an optical power meter. After the initial readings taken on short length of test cable, a second similar reading is taken with the installed cable place. The difference between the two readings indicates the additional power losses due to fiber length differences in optical qualities of connectors. Because approximate fiber losses are known, losses greater than $1.0 \mathrm{~dB}$ to $1.5 \mathrm{~dB}$ above fiber losses might indicate an inferior connection-requiring either re-polishing or replacement.

Optical Power meter:Power meters often read directly in power units such as dBM and $\mathrm{dB} \mu$. By using connector adapters and light sources of the same wavelength as installed equipment, an accurate measure of link losses with connectors and splices may be obtained.

\subsection{Optical Time Domain Reflectometer (Otdr)}

OTDRs are typically used to measure distance and attenuation over the entire fiber link. They are also used to identify specific points along the link where losses occur, such as splices. An OTDR is optical radar which measures time of travel and return strength of a short pulse of light launched into an optical fiber. Small reflections occur throughout the fiber, becoming weaker as power levels drop distance. At major breaks, large reflections occur and appear as strong peaks on oscilloscopes. Testing of short and medium distance fiber optic systems seldom requires an OTDR. In smaller systems, optical power meter tests are faster and more useful. Many instrument rental companies are now offering OTDR as well as other fiber optic splicing and test equipment.

\subsection{Inspection Methods And Tools}

ADSS aerial cables require special safety precautions as a result of their possible semi- conducting nature. Special safety procedures and arrangements need to be defined to inspect ADSS aerial cables whenever working under live line conditions. While ADSS cables can be considered non-conducting during the installation in the presence of live lines (i.e. if regulations allow), ADSS is very likely to become semiconductive during the service life as a result of surface deposits and the hydrophilic nature of the jacket material, which should be considered during maintenance. Equipment required for inspection and maintenance for ADSS, OPGW, and WRAP aerial cables and wires is usually limited to technology that can be used to check for the integrity and degradation of the transmission in the fiber of the communication cable. Currently, there are no inspection tools (i.e., other than close-up visual examination)

\section{Conclusions}

This paper, discussed an Optical Fibre cable in domestic communication of power grid network as an alternative media installed to the power line carrier and microwave radio to enhance adequate and efficient communication and security in the grid. The use of $132 \mathrm{KV}$ power transmission line infrastructures between the Optical network can satisfy present need of high speed and reliable data, video and voice communications from all existing equipments, offices, operators, and maintenance engineers and technicians. Primarily, there are three different cable options available to an electric companies that opts to integrate communications into their existing power transmission system. The currently available cables and wires that can be used in high voltage passages are:

ADSS (All-Dielectric Self Supporting Cable)

OPGW (Optical Ground Wire)

WRAP (Optical Cable Wrapped on Ground Wire)

Future expansion of the network to include all other $132 \mathrm{KV}$ and $400 \mathrm{KV}$ substations have been taken into account in the design work. Extra Optical Fibres in the OPGW are reserved for future use to connect all towns the network pass through or pass by. And the people living in these towns will be served when the future expansions are implemented. The issue of security power network Inspection and damage assessment tools need to be identified to evaluate the integrity and confidentiality of aerial cables and wires. These inspection and assessment tools are required by electric utilities to locate developing problem areas prior to catastrophic failure. Regular inspection and reliable damage detection procedures and tools could be used in preventive maintenance programs that could significantly reduce repair cost and increase the reliability of the communication system. 


\section{References}

[1]. M. N. Ott, R. Switzer, R.F. Chuska, F. V. LaRocca, W. J. Thomes, L. W. Day, and S. L. Macmurphy, "The Optical Fiber Array Bundle Assemblies for the NASA Lunar Reconnaissance Orbiter; Evaluation Lessons Learned for Flight Implementation," invited paper, ICSO, October 2008.

[2]. X. L. Jin, M. N. Ott, F. V. LaRocca, R. F. Chuska, S. M. Schmidt, A. J. Matuzseski, R. Switzer, W. J.Thomes, and S. L. Macmurphy, "Space Flight Qualification on a Novel Five-fiber Array Assembly for the Lunar Orbiter Laser Altimeter (LOLA) at NASA GSFC," SPIE, Vol. 6713, Aug 2007.

[3]. R. Setchell, K. Meeks, W. Trott, P. Klinsporn, and D. Berry, "High-Power Transmission Through Step-Index Multimode Fibers," SPIE, Vol. 1441, p. 61, 1991.

[4]. R. Setchell, “An Optimized Fiber Delivery System for Q-switched, Nd:YAG Lasers,” SPIE, Vol. 2296, p.608, 1997.

[5]. W. Sweatt and M. Farn, "Kinoform/Lens System for Injecting a High Power Laser Beam Into an Optical Fiber," SPIE, Vol. 2114, p. 82, 1994.

[6]. R. Setchell, "Damage Studies in High-Power Fiber Transmission Systems," SPIE, Vol. 2114, p. 87, 1994.

[7]. W. Sweatt, R. Setchell, M. Warren, and M. Farn, "Injecting a Pulsed YAG Laser Beam Into a Fiber,"SPIE, Vol. 3010, p. 266, 1997.

[8]. R. Setchell, "Laser Injection Optics for High-Intensity Transmission in Multimode Fibers,” SPIE, Vol. 4095, p. 74, 2000.

[9]. L. Weichman, F. Dickey, and R. Shagam, "Beam Shaping Element for Compact Fiber Injection Systems,"SPIE, Vol. 3929, p. $176,2000$.

[10]. F. Dickey, S. Holswade, Laser Beam Shaping: Theory and Techniques, Chapter 7 (D. Brown, F. Dickey,

[11]. L. Weichman, Multi-aperture Beam Integration Systems), Marcel Dekker, Inc., 2000: pp. 273-311.

[12]. Carter, “Arc Control Devices For Use On All- Dielectric Self Supporting Optical Cables”, IEE Proceedings-A, IEE, Vol 140, No 5, pp. 357-361, September 1993.

[13]. Rowland and Easthope, "Electrical Aging and Testing of Dielectric Self- supporting Cables for Overhead Power Lines", IEE Proceedings-A, IEE, Vol 140, No 5, pp. 351-356, 1993.

[14]. Carter and Waldron, "Mathematical Model of Dry Band Arcing on Self-Supporting All-Dielectric Optical Cables Strung on Overhead Power Lines", IEE Proceedings-A, IEE, Vol 139, No 3, pp. 185-196, May 1992.

[15]. Electrostatic and Electromagnetic Effects of Ultrahigh Voltage Transmission Lines, Electric Power Research Institute, Palo Alto, Final Report, EPRI El-802, Project 566, June 1978.

[16]. Billings, and Smith, "Tracking in Polymeric Insulation”, IEEE Transaction on Electrical Insulation, IEEE, New York, Vol EI-2, No 3, pp. 131-137, Dec 1967.

[17]. Loberg, and Salthouse, "Dry Band Growth on Polluted Insulation", IEEE Transaction on Electrical Insulation, IEEE, New York, NY, Vol EI-6, No 3, pp. 136- 141, September 1971.

[18]. (17) Stimper, and Middendorf, "Mechanisms of Deterioration of Electrical Insulation Surfaces", IEEE Transactions on Electrical Insulation, IEEE, New York, NY, Vol EI- 19, No 4, pp. 314-320, August 1984.

[19]. Clabburn, Penneck, and Swinmurn, "The Outdoor Performance of Plastic Materials Used as Cable Accessories", IEEE Proceedings, IEEE, New York, NY,pp. 1833-1842, 1973.

[20]. Astle-Fletcher, "Proposal For Preventing Damage to High-Voltage Plastics Insulators by Surface Electrical Discharges", Correspondence, British Railways Department, Derby, U.K., 1969.

[21]. Austin, Barnett, and Gaylard, “Optical Communications Using Overhead Transmission Lines”, CIGRE, TC 35-04, pp. 1-7, 1984.

[22]. Burggendieck, Herff, and Mainka, "25Km Optical Aerial Cable Link on 100Kv Overhead Line", CIGRE, TC 35-09, pp. 1-8, 1984 .

[23]. Gaylard, Dey, Holden, Taylor, Smith, Maddock, and Kent, "Optical Communication Using Overhead Power Lines”, CIGRE , TC 35-01, pp. 1-11, 1982 .

[24]. Jurdens, Haag, and Buchwald, "Experience with Optical Fibre Aerial Cables on High Voltage Power Lines", CIGRE , TC 22-11, pp. $1-10,1988$

[25]. Kouteynikoff, Pays, Mollier, Pannier, Bonicel, and Trombert, "Optical Fiber Communication on the Electricity Transmission Network", CIGRE, TC 35-103, pp. 1-10, 1990.

[26]. Chung, and Ding, "Stress Strain Characteristics of Self Supporting Aerial Optical Fibre Cables", IWCS Proceedings, IWCS, pp. 178-185, 1991.

[27]. Olszewki, and Masterson, "Lightning Considerations in Optical Cables Design”, IWCS Proceedings, IWCS, pp. 141-147, 1986.

[28]. Takahara, Ishihata, Sairo, Niikura, Horima, Kurosawa, Takeda, and Ohmori "'Withstanding High Voltage Characteristics of Non Metallic Self Supporting Optical Cable", IWCS Proceedings, IWCS, pp. 441-448, 1987.

[29]. X. L. Jin, M. N. Ott, F. V. LaRocca, R. F. Chuska, S. M. Schmidt, A. J. Matuzseski, R. Switzer, W. J. Thomes, and S. L. Macmurphy, "Space Flight Qualification on a Novel Five-fiber Array Assembly for the Lunar Orbiter Laser Altimeter (LOLA) at NASA GSFC," SPIE, Vol. 6713, Aug 2007.

[30]. R. Setchell, K. Meeks, W. Trott, P. Klinsporn, and D. Berry, "High-Power Transmission Through Step- Index Multimode Fibers," SPIE, Vol. 1441, p. 61, 1991.

[31]. R. Setchell, “An Optimized Fiber Delivery System for Q-switched, Nd:YAG Lasers,” SPIE, Vol. 2296, p. 608, 1997.

[32]. W. Sweatt and M. Farn, "Kinoform/Lens System for Injecting a High Power Laser Beam Into an Optical Fiber," SPIE, Vol. 2114 , p. 82, 1994.

[33]. R. Setchell, "Damage Studies in High-Power Fiber Transmission Systems," SPIE, Vol. 2114, p. 87, 1994.

[34]. C.N. Carter, J. Deas, N.R. Haigh, and S.M. Rowland, “Applicability of all-dielectric self supporting cable system to very high voltage overhead power lines," Proceeding of the 46th International Wire and Cable Symposium, 1997, pp. 622-631.

[35]. (34) K.S. Edwards, P.D. Pedrow, R.G. Olsen, "Portable ADSS surface contamination meter," 1999 Annual Report Conference on Electrical Insulation and Dielectric Phenomena, Piscataway, NJ, USA; IEEE Cat. No. 99CH36319, Vol.1, 1999, pp. 158-61.

[36]. J.C.G. Wheeler, M.L. Lissenburg, J.D.S. Hinchliffe, M.E. Slevin, "The development and testing of a track resistant sheathing material for aerial fiber cables," Proceedings of the 5th DMMA, Canterbury, June 1988, IEE Conference publication No. 286, pp. 69-72.

[37]. S.M. Rowland, C.N. Carter, "The evaluation of sheathing material for an all dielectric self-supporting communication cable, for use on long span, overhead power lines," 5th International Conference on Dielectric Materials, Measurements and Applications, London, UK; IEE Conference publication No. 289, 1988; pp.p.77-80

[38]. A.G.W.M. Berkers, J.M. Wetzer, "Electrical stress on self-supporting metal-free optical cable in high voltage networks," Proceedings for the 5th DMMA, Canterbury, June 1988, IEE Conference publication No 286, pp. 69-72. 